Cuad. Soc. Esp. Cienc. For. 46(1): 1-16 (2020)

Doi: https://doi.org/10.31167/csecfv0i46.19895

Sociedad Española

\title{
Estudio de la presencia de defoliadores tempranos de Quercus suber en árboles con distinto perfil de emisión de monoterpenos foliares
}

Study of the Early defoliators of Quercus suber trees with different monoterpene foliar emission profile

\author{
Robles, D. ${ }^{1} ;$ Sánchez-Osorio, I. ${ }^{*}$; Tapias, R. ${ }^{1}$ \\ 'Departamento de Ciencias Agroforestales, Escuela Técnica Superior de Ingeniería, \\ Universidad de Huelva, 21007. Campus El Carmen (Huelva). \\ Teléfono: +34959217635/Fax: +34959217560 \\ *Autor para correspondencia: isanchez@uhu.es
}




\section{Resumen}

En este trabajo se estudió la presencia de insectos defoliadores de hoja tierna (dentro de los órdenes Lepidoptera e Hymenoptera), en una dehesa de Quercus suber en Huelva (TM Almonte), y su relación con los perfiles de emisión foliar de monoterpenos de los árboles. Los muestreos consistieron en vareos semanales (15 golpes por árbol y vareo) entre el 28/3/2019 y el 10/5/2019 (comprendiendo el vuelo de las principales especies en la zona estudiada). Se estudiaron 25 alcornoques cuyo perfil de emisión de monoterpenos foliares era conocido previamente (12 con perfil tipo pineno, 13 con perfil tipo limoneno). Se identificaron un total de 272 larvas pertenecientes a 9 especies. Las especies dominantes fueron Catocala nymphagoga (40.8\%) y Periclista andrei (27.6\%); junto a ellas se encontraron Bena bicolorana, Syntaurucus pirithous, Drepana uncinula, Archyps xylosteana, Cyclophora punctaria, Satyrium esculi y Lasiocampa trifolii. El 70.6\% de las larvas se encontró en árboles con perfil tipo pineno, el 29.4\% restante en árboles de perfil tipo limoneno. La composición en especies resultó diferente entre árboles de distinto perfil emisor. Mientras que L. trifolii y $S$. pirithous no se encontraron en árboles de perfil limoneno (escasas en tipo pineno), D. uncinula y A. xylosteanus tuvieron abundancia ligeramente mayor para el perfil tipo limoneno. La presencia conjunta de las cuatro especies más relevantes (C. nymphagoga, P. andrei, B. bicolorana y $C$. punctaria) resultó un $62.7 \%$ menor en árboles con perfil tipo limoneno que en árboles de tipo pineno.

Palabras clave: Lepidoptera, Hymenoptera, alcornoque, Catocala, Periclista, Bena.

\section{Summary}

The presence of soft-leaf defoliating insects (within orders Lepidoptera and Hymenoptera), in a Quercus suber dehesa in Huelva (Municipality of Almonte), and their relationship with the leaf monoterpene emission profiles was studied. Twenty five cork oaks having a known monoterpene emission profile (12 with pinene-type profile; 13 with limonene-type profile), were sampled to determine early defoliator's larvae presence. Larvae were collected weekly, by rapping tree branches sharply with a beating stick ( 15 beating per tree per sampling event), between 3/28/2019 and 5/10/2019 (comprising the whole larval development period). A total of 272 larvae belonging to 9 species were identified. The dominant species were Catocala nymphagoga (40.8\%) and Periclista andrei (27.6\%); Bena bicolorana, Syntaurucus pirithous, Drepana uncinula, Archyps xylosteana, Cyclophora punctaria, Satyrium esculi and Lasiocampa trifolii were also encountered. A 70.6\% of larvae were found in trees with pinene-type profile, whiles a $29.4 \%$ were found in trees having limonene-type profile. The species composition was different between trees having different emission profiles. While L. trifolii and S. pirithous were not found in limonene profile trees (scarce in pinene-type), D. uncinula and A. xylosteanus had slightly greater abundance in trees with limonene-type profile. The joint presence of the four most relevant species (C. nymphagoga, P. andrei, B. bicolorana and C. punctaria) was $62.7 \%$ lower in trees with a limonene profile than found in trees having pinene-type profile.

Keywords: Lepidoptera, Hymenoptera, cork oak, Catocala, Periclista, Bena. 


\section{Introducción}

Las dehesas se caracterizan por su complejidad ecológica y económica, que refleja la diversidad biológica y ambiental de los agrobiosistemas mediterráneos (San Miguel, 1994); y han permitido durante siglos el desarrollo social de ciertas zonas tradicionalmente desfavorecidas. El alcornoque, Quercus suber L. es la segunda especie en importancia en las dehesas de la zona suroccidental (Alejano et al., 2011). Los insectos defoliadores son componentes importantes de los ecosistemas forestales: afectan al crecimiento de los árboles e influyen en el ciclo de los nutrientes, proporcionan alimentos a niveles tróficos superiores y algunas especies contribuyen a la polinización como adultos (Summerville and Marquis, 2016). Entre los defoliadores que afectan al género Quercus se han citado 60 especies de Lepidoptera (en 14 familias, la mayoría de Noctuidae), un himenóptero (Periclista andrei Konow), los géneros Coeliodes Shoenherr y Brachyderes Shoenherr (Coleoptera) (Antonietty, 2013 y autores citados allí). Los efectos de los defoliadores van desde la afección de la floración y foliación de los árboles, con la merma de la producción de fruto y madera, hasta alteraciones de su fisiología (Magnoler and Cambini, 1970; Luciano and Roversi, 2001). Un buen número de especies defoliadoras se mantienen generalmente en bajos niveles poblacionales, provocando daños en caso de explosión demográfica ocasional, como ocurre con Catocala nymphagoga Esper (Toimil, 1987; Monreal et al., Tiberi et al., 2016). Pero algunas especies suelen producir daños con frecuencia, como Lymantria dispar L., Tortrix viridana L. o el himenóptero P. andrei en el Mediterráneo occidental (Branco and Ramos, 2009; Pereira et al., 2012; Antonietty, 2013; Tiberi et al., 2016). Así, defoliaciones de T. viridana mantenidas durante dos años llegan a reducir el crecimiento del árbol hasta en un 70\% (Teodorescu and Simionescu, 1994), habiéndose valorado la pérdida de bellota bajo la acción de este tortrícido en más de $500 \mathrm{~kg} \mathrm{ha}^{-1}$ año $^{-1}$ (Torrent, 1963). La ocurrencia de defoliaciones severas y continuas se considera un factor desencadenante en el decaimiento del arbolado, predisponiendo a los árboles al ataque de otros organismos, como patógenos o insectos xilófagos (Thomas, 2008; Tiberi et al., 2016).

Los terpenoides y otros compuestos orgánicos volátiles (COVs) desempeñan un papel importante tanto en la fisiología de las plantas como en las interacciones de las plantas con su entorno; por ejemplo, protegiendo las plantas contra los factores de estrés, actuando como un mecanismo para la comunicación entre individuos o incluso atrayendo a predadores y parasitoides de herbívoros (Dicke and Baldwin, 2010; Grote et al., 2013). El género Quercus se encuentra entre los mayores emisores de COVs (Pearse et al., 2013), considerándose $Q$. suber como un fuerte emisor de monoterpenos (Pio et al., 2005; Lavoir et al., 2011). El efecto que la emisión de compuestos foliares tiene sobre los insectos defoliadores puede ser de distinta índole. Así, pueden actuar como señales para la selección intraespecífica de ciertos hospedantes individuales, o como repelentes si son emitidos específicamente por una planta afectada por herbivoría (Gols, 2015). Se ha sugerido que COVs como $\alpha$-pineno, $\beta$-pineno y 1 -hexanol pueden resultar atrayentes de L. dispar hacia Larix gmelinii Rupr. (Li et al., 2012), y que los terpenoides podrían influir en los patrones de herbivoría de L. dispar en Quercus ilex L. (Solla et al., 2016). 
Este estudio pretende contribuir al conocimiento de los mecanismos que condicionan la composición en especies defoliadoras (Lepidoptera e Hymenoptera) en $Q$. suber, en particular la relación entre la presencia y abundancia de larvas defoliadoras de hoja tierna y la tipología de emisión de monoterpenos foliares. Los objetivos planteados son:

1. Determinar las especies defoliadoras de hoja tierna presentes en pies de $Q$. suber de tipología de emisión foliar conocida.

2. Comparar la composición en especies defoliadoras entre grupos de árboles de distinto perfil emisor.

3. Comparar la actividad herbívora en campo entre grupos de árboles de distinto perfil de emisión foliar.

\section{Material y Métodos}

\section{1. Árboles estudiados}

El estudio se realizó en 2019 en la dehesa San Enrique (Almonte, Huelva. $\left.37^{\circ} 15^{\prime} 43.73^{\prime \prime} \mathrm{N}, 6^{\circ} 28^{\prime} 34.65^{\prime \prime} \mathrm{O} ; 80 \mathrm{~m} \mathrm{snm}\right)$. Esta dehesa está poblada mayoritariamente por Q. suber (densidad de 75 árboles ha ${ }^{-1}$ ), con pies dispersos de Quercus ilex L. y Pinus pinea L., y escasa cobertura de matorral por la realización de gradeos anuales. Se lleva a cabo aprovechamiento corchero y, de forma intermitente, del pasto. En general, el vigor del arbolado es deficiente, observándose una fuerte incidencia del cerambícido xilófago Cerambyx welensii Küster (López-Pantoja et al., 2008).

Estudios anteriores sobre la incidencia de $C$. welensii en esta dehesa permitieron conocer el perfil de emisión foliar de monoterpenos de un grupo de árboles (Sánchez-Osorio et al., 2019). El procedimiento de muestreo, resumido, consistió en capturar, con la ayuda de una bomba de vacío (SP200 EC-LC. Schwarzer Präzision, Essen, Alemania. Caudal de $120 \mathrm{ml} \mathrm{min}^{-1}$ durante 5 minutos), los COVs liberados por ramas introducidas en cámaras de encerramiento dinámico (poliéster, $38 \times 25$ cm. Albal. Cofresco, Madrid). El aire extraído de cada cámara atravesó un tubo de vidrio con $150 \mathrm{mg}$ de Tenax TA (Tubo Orbo, 60/80 mesh. Supelco, Madrid), donde los COVs quedaron adsorbidos; simultáneamente, y en las mismas condiciones experimentales, se muestreó el aire en el exterior de la cámara de encerramiento. Las muestras se analizaron posteriormente mediante cromatografía de gases unida a espectrometría de masas.

Se seleccionaron, así, 25 alcornoques de perfil de emisión conocida (Fig. 1): 13 presentando perfil tipo limoneno (presencia relativa de limoneno $>30 \%$ de la emisión total de monoterpenos. Staudt et al., 2004), y 12 ejemplares con perfil dominado por compuestos de tipo pineno ( $\alpha$-pineno, $\beta$-pineno y sabineno). 


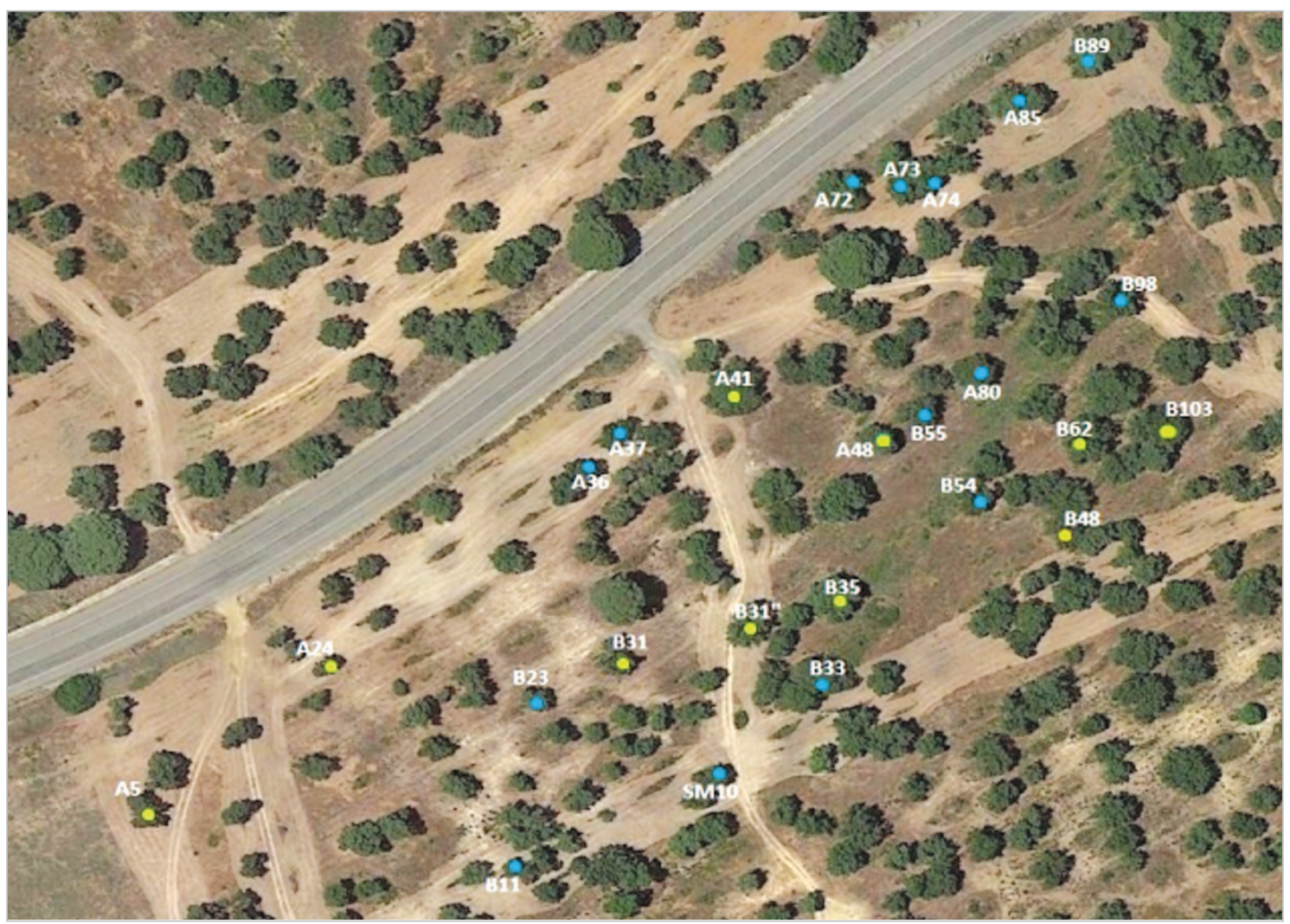

Figura 1. Ejemplares de Quercus suber en los que se estudió la presencia de defoliadores tempranos. Puntos verdes indican los pies de perfil emisor tipo pineno; puntos azules señalan los árboles de perfil emisor tipo limoneno.

Dehesa San Enrique (TM Almonte, Huelva).

\subsection{Muestreo de defoliadores}

El muestreo se realizó mediante vareos semanales, entre el 28 de marzo y el 10 de mayo. Este período abarcó el desarrollo larvario de los defoliadores tempranos. La temperatura media osciló entre $15^{\circ} \mathrm{C}$ (las cuatro primeras semanas del muestreo) y $18{ }^{\circ} \mathrm{C}$ (las dos últimas semanas). Se registraron 14 días de precipitación durante el período de muestreo, con máximo de $13 \mathrm{~mm}$ y media de $4.6 \mathrm{~mm}$ (Observatorio de Almonte, "Bodegones").

Cada vareo comprendió 15 golpes por árbol (Granados et al., 2000), con pértiga telescópica de aluminio de 3.60 metros de longitud. En cada muestreo se varearon, siguiendo el mismo recorrido, los 25 árboles. Las larvas se recolectaron en una lona de plástico ( 4 × $4 \mathrm{~m}$ ) extendida bajo la copa (orientación sur y cubriendo $1 / 3$ de su proyección). Una vez identificadas las especies y anotado el número de individuos, todas las larvas recolectadas se mantuvieron en laboratorio en un terrario de plástico de 50 x $30 \mathrm{~cm}$ y $30 \mathrm{~cm}$ de alto, provisto de ramillos con hojas tiernas de alcornoque, bajo condiciones ambientales $\left(20^{\circ} \mathrm{C}\right.$; $\left.70 \% \mathrm{HR}\right)$. 


\subsection{Estimación de defoliación en campo}

El nivel de defoliación en campo se estimó recolectando, al término del período de muestreo, 3 ramillos por árbol, de $30 \mathrm{~cm}$ de longitud, situados en orientación sur a una altura de 1.70 metros y espaciados 1 metro. En cada ramillo se contó el número total de hojas y el número de hojas que mostraron roeduras; el software ImageJ permitió medir su área, longitud y anchura en la zona más amplia del limbo a partir de fotografías. Para la estimación del grado de afección por herbivoría en cada hoja, se emplearon dos rectas de regresión obtenidas a partir de 60 hojas intactas (rango medido: 0.5 a $6.7 \mathrm{~cm}^{2}$; media de $3.7 \mathrm{~cm}^{2}$ ): por un lado para el área de la hoja en función de su anchura $\left(\mathrm{R}^{2}=0.81\right)$, y por otra parte para el área en función del producto longitud*anchura $\left(\mathrm{R}^{2}=0.97\right)$. El daño por herbivoría en cada hoja se computó como la pérdida de superficie $(\%)$ de cada hoja tras comparar el área medida con el área estimada a partir de las regresiones (cuando fue posible, se empleó la regresión para el área respecto a longitud*anchura).

\subsection{Análisis estadístico}

Las capturas por árbol, así como los porcentajes de daño de las hoja, se analizaron mediante el test robusto de Yuen-Welch para medias recortadas $\left(\mathrm{Me}_{0.2}\right.$, media aritmética obtenida después de eliminar el 20\% de los valores más altos y el $20 \%$ de los valores más bajos en cada muestra de partida). Se buscó, así, reducir el posible efecto distorsionador de valores extremos de capturas o daños en algunos árboles. El análisis de los daños por hoja para diferentes percentiles se realizó mediante el test de Brown-Mood.

Para analizar la composición de especies defoliadoras y su relación con los perfiles emisores de $Q$. suber se empleó un escalamiento dimensional no métrico (NMDS. Distancias de Chord), seguido de un análisis de varianza permutacional multivariado (PERMANOVA. Distancia de Chord; 999 permutaciones), comprobando la dispersión multivariante (paquete vegan; Oksanen et al., 2015). Como variables clasificadoras del PERMANOVA se emplearon el "tipo" de perfil emisor y la "ubicación" de los árboles en la masa. La ubicación de los árboles se incluyó tras observar en campo (Fig. 1) que mientras la mayoría de pies presentaban alrededor espacios de cierta amplitud libres de obstáculos (en general, de extensión al menos dos veces el diámetro de la proyección vertical de su copa y según al menos dos orientaciones distintas), tres árboles se encontraban más claramente rodeados de pies vecinos (pies A73, A80, B31". Fig. 1). Se consideró que esta circunstancia podría tener efecto en la localización de hospedantes por insectos voladores. Los análisis estadísticos se realizaron con el programa $R$, versión 3.1.0, utilizando $\alpha=0.05$ como nivel de significación. 


\section{Resultados}

\subsection{Especies defoliadoras}

Se recolectó un total de 272 larvas pertenecientes a 9 especies (8 lepidópteros y 1 himenóptero), incluidas en 7 familias (Tabla 1). Es de destacar que en 7 de los 25 pies ( 3 de perfil pineno y 4 de perfil limoneno) no se obtuvo captura de larva alguna. Indicaremos que se encontraron 15 ejemplares de Cryphia algae F., especie que se nutre de líquenes y no se ha computado de cara al presente estudio. Las especies defoliadoras tempranas más abundantes fueron $C$. nymphagoga $(40.8 \%)$ y $P$. andrei (27.6\%). El 93.4\% de los ejemplares pertenecieron en conjunto a las especies $C$. nymphagoga, $P$. andrei, $C$. punctaria y B. bicolorana.

Tabla 1. Especies defoliadoras (Lepidoptera, excepto la familia Tenthredinidae de Hymenoptera) de hoja tierna de Quercus suber en la dehesa San Enrique (TM Almonte, Huelva). Se indica la relevancia porcentual y la abreviatura usada en la fig. 3.

\begin{tabular}{cccc}
\hline FAMILIA & ESPECIE & ABREVIATURA & $(\mathbf{\%})$ \\
\hline Noctuidae & Catocala nymphagoga Esper & Catnym & 40.8 \\
& Bena bicolorana L. & Benbic & 11.0 \\
\hline Lycaenidae & Syntaurucus pirithous L. & Synpir & 0.7 \\
& Satyrium esculi Hb. & Satesc & 1.1 \\
\hline Tenthredinidae & Periclista andrei Konow. & Perand & 27.6 \\
Tortricidae & Archips xylosteana L. & Arcxyl & 2.2 \\
Drepanidae & Drepana uncinula Bkh. & Dreunc & 2.2 \\
Lasiocampidae & Lasiocampa trifolii D. \& S. & Lastri & 0.4 \\
Geometridae & Cyclophora punctaria L. & Cycpun & 14.0 \\
\hline
\end{tabular}

La evolución temporal de capturas se muestra en la fig. $2 a$. El pico de presencia más temprano correspondió a B. bicolorana y A. xylosteana (en torno al $17 \mathrm{de}$ abril); $C$. nymphagoga y $P$. andrei presentaron similar pico temporal de capturas (sobre el 25 de abril); la especie más tardía fue C. punctaria (máximo en torno al 3 de mayo).

\subsection{Relación entre la presencia de defoliadores y el perfil de emisión de los árboles}

E1 70.6\% de las larvas se encontró en árboles con perfil tipo pineno (_-Cuadrado $=46.12, \mathrm{P}<0.001)$. Las capturas medias por árbol $\left(\mathrm{Me}_{0.2} \pm\right.$ Error $\left._{0.2}\right)$ fueron mayores en pies de perfil tipo pineno $(12.14 \pm 5.06)$ que en árboles de perfil tipo limoneno $(5.83 \pm 0.97)$, pero no significativamente (Yuen-Welch: $\mathrm{P}=0.167)$. La evolución de capturas fue similar en árboles de los dos tipos emisores (Fig. 2b), con un claro incremento en torno al 3 de abril, un primer pico de capturas el 17 de abril y el máximo de capturas el 25 de abril. 

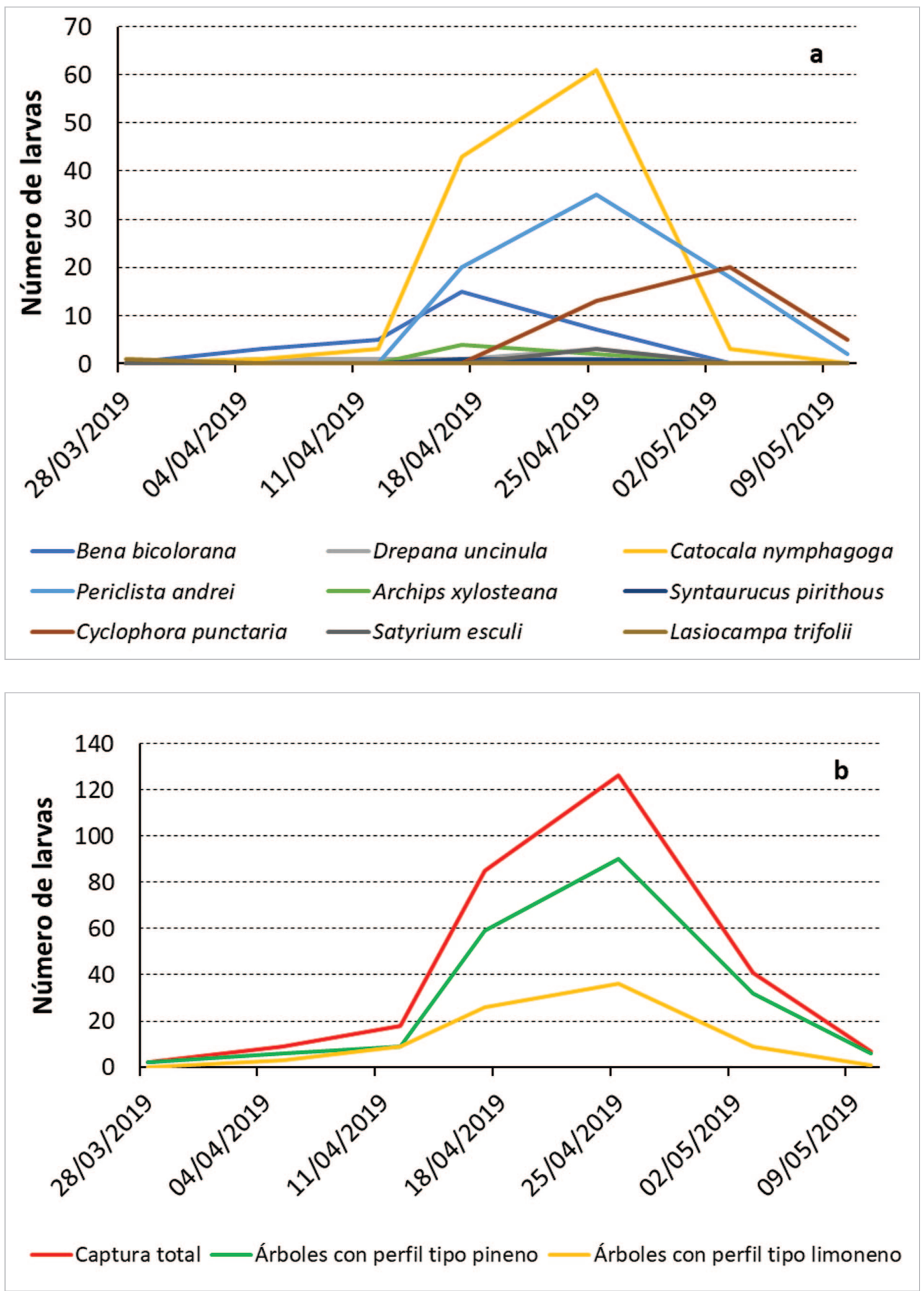

Figura 2. Evolución temporal de capturas, por especie y tipo emisor, de defoliadores de hoja tierna de Quercus suber en la dehesa San Enrique (TM Almonte, Huelva). 
El análisis exploratorio NMDS (Fig. 3) sugirió la presencia de dos gradientes, uno para el reparto de especies y otro para el reparto de árboles de cada tipo emisor. Los elipsoides representativos de los dos grupos de árboles sugieren que el reparto de árboles de perfil emisor tipo limoneno se asociaría al eje determinado entre las especies $C$. nymphagoga, A. xylosteana y B. bicolorana; los pies de perfil tipo pineno se asociarían al eje determinado por $C$. nymphagoga, . andrei y dos especies con aparición testimonial como $S$. pirithous y L. trifolii. El análisis de varianza multivariado reveló diferencias marginalmente significativas entre la composición en especies para árboles de distinto perfil emisor (PERMANOVA: $F=2.20, P=$ 0.066) que estuvieron influidas por la ubicación de los árboles (PERMANOVA: $F$ $=3.51, P=0.016$ para la interacción ubicación * tipo). Para el conjunto de árboles con ubicación próxima a bordes o grandes rasos $(\mathrm{N}=14)$, la diferencia en composición de especies según la tipología de emisión de los árboles resultó más clara (PERMANOVA: $F=2.76, P=0.035$ ).

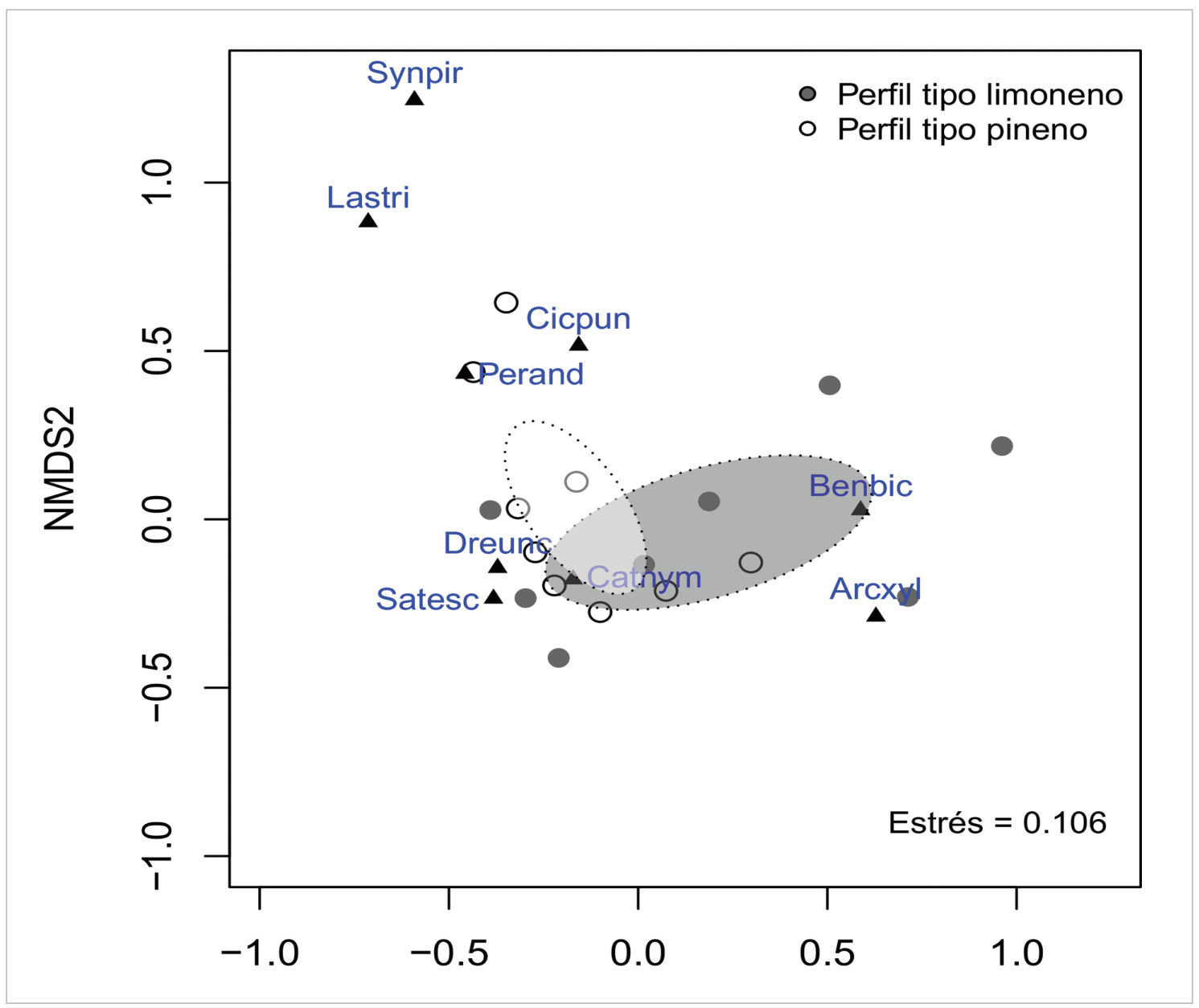

Figura 3. Escalamiento multidimensional no métrico (Distancia de Chord) para la distribución de especies defoliadoras temparanas (triángulos) y árboles (círculos) con distinto perfil emisor de monoterpenos foliares. Las especies se nombran de forma abreviada (ver Tabla 1). Los elipsoides muestran el intervalo de confianza (95\%, a partir del error estándar) para los centroides de cada grupo de árboles. 


\subsection{Relación entre los daños por defoliación y el perfil de emisión de los árboles}

Las hojas de árboles con perfil emisor tipo pineno fueron un 13\% más cortas y un $17.5 \%$ más estrechas que las recolectadas en árboles de tipo limoneno (YuenWelch: $t=8.72$ y $t=6.72$ respectivamente. $P<0.001)$. El número de hojas $\left(\mathrm{Me}_{0.2} \pm\right.$ Error $_{0.2}$ ) con incidencia por herbivoría en campo en los tres ramillos de cada árbol resultó mayor en pies de perfil tipo limoneno (39.6 \pm 1.65 hojas) que en árboles de perfil tipo pineno (25.9 \pm 4.84 hojas.) (Yuen-Welch: $t=3.39, P=0.01)$. Sin embargo, el grado de afección promedio de las hojas fue ligeramente mayor en árboles con perfil tipo pineno (13.73\%) que en árboles con perfil tipo limoneno (11.98\%), resultando significativamente mayor el daño en perfil tipo pineno tanto para los menores niveles de afección (percentil 10\%) como para los mayores niveles de afección (percentil 85\%) (Brown-Mood: $P=0.044$ y $P=0.021$, respectivamente).

\section{Discusión}

Dos especies dominaron claramente en los muestreos del presente estudio: $C$. nymphagoga y $P$. andrei. Mientras la primera especie ya ha sido citada como potencialmente causante de daños de relevancia en Quercus (Monreal et al., 1992; Tiberi et al., 2016), P. andrei se ha venido considerando una especie con presencia muy local y comportamiento marcadamente epidémico (Pereira et al., 2014, Tiberi et al., 2016). Otras especies encontradas en este trabajo también se han considerado relevantes en Quercus, en especial en $Q$. ilex y Q. suber, como A. xylosteana (Toimil, 1987; Granados et al., 2000) o D. uncinula y S. esculi (Toimil, 1987; Monreal et al., 1992). La primera cuestión llamativa resultó la ausencia de larvas de T. viridana, lo cual ha sido descrito ya para $Q$. suber (Extremera et al., 2004). La sincronización entre el desarrollo larvario y la fenología del hospedante resulta un factor crítico para esta especie (Ivashov et al., 2002; Antonietty, 2013), pudiendo resultar el período de brotación en alcornoque, más tardío que en la encina, inadecuado para T. viridana en algunas localizaciones. En escenarios de baja presencia de T. viridana, la acción de esta especie suele ser reemplazada por otros tortrícidos, como el género Archips (Sánchez-Herrera y Soria, 1987)

La selección de hospedantes resulta crucial para que los insectos fitófagos cubran sus requerimientos nutricionales y encuentren sitios de oviposición; los COVs de plantas desempeñan un papel importante en este proceso (Carrasco et al., 2015; Conchou et al., 2019). A su vez, la herbivoría altera el fenotipo de la planta hospedante, lo que modula la capacidad de elección y utilización del hospedante por la misma especie incidente u otros herbívoros (Srinivasan, 2019; y autores citados allí). La inducción de la emisión de COVs por ataques de herbívoros es un tema extensamente tratado, pero son más limitados los estudios sobre la divergencia intraespecífica en la producción de COV inducida por estrés y su relación con la defensa de las plantas frente a sus atacantes (Staudt et al., 2010).

El número de larvas recolectadas en el presente estudio ha sido moderado, quizá 
debido a un bajo nivel poblacional general del complejo defoliador o al método de muestreo seguido. Aun considerando, por ello, los resultados desde un enfoque preliminar, es destacable que tanto los niveles de captura globales como el análisis de la composición en especies hayan mostrado diferencias entre pies de $Q$. suber con distinto perfil de emisión foliar. Así, la tipología de emisión foliar de $Q$. suber, ampliamente descrita (Staudt et al., 2004; Pio et al., 2005; Lavoir et al., 2011; SánchezOsorio et al., 2019), podría influir en la localización intraespecífica preferencial de hospedantes por insectos defoliadores. Se ha citado para los metabolitos secundarios de las plantas, como los terpenos y fenoles, distintos efectos en los insectos defoliadores: mientras que en general se les atribuye acción repelente (Kneeshaw et al., 2015), algunos COVs, como los monoterpenos de coníferas, pueden tener tanto dicho efecto (caso del limoneno) como actividad potencial como precursores nutricionales feromonales (caso del $\alpha$-pineno) (Bowsher et al., 2008). La emisión de COVs inducida por la herbivoría puede, además, ser usada por organismos de distintos niveles tróficos, en una relación dosis-respuesta; por ejemplo, causando atracción de enemigos naturales de insectos fitófagos (Kighati et al., 2009; Gols, 2012).

Árboles de distinto perfil emisor han mostrado en el presente estudio una morfología foliar diferente, además de una distinta extensión de la incidencia por herbivoría y un diferente grado de daño en las hojas. Esto resulta interesante si se considera que el perfil de emisión foliar en $Q$. suber tiene una determinación genética (Staudt et al., 2004). Para T. viridana se ha citado que la estimulación visual podría desempeñar un papel determinante en la localización de alimento por las larvas de estadios avanzados (Antonietty, 2013). La calidad del alimento no solo influye en el desarrollo larvario, sino que puede tener relevancia en la actividad feromonal. Es interesante la revisión a este respecto de Henneken et al., (2017), que citan tres formas en las que tal relación se lleva a cabo:

1. Una dieta de calidad propicia una producción feromonal de calidad (por tanto una señal sexual potente).

2. La dieta puede aportar precursores feromonales no sintetizables de novo por el insecto.

3. La dieta puede influir en la propia tipología de la firma feromonal.

Entre las especies encontradas en este estudio, solo se ha citado hasta la fecha (ver Ando, 2019) feromona sexual para A. xylosteana (los acetatos de tetradecilo Z11 y E11) (Ando et al., 1978; Frerot et al., 1983). Se ha citado, así mismo, actividad feromonal en otras especies emparentadas a las encontradas en este estudio, como Drepana bilineata Packard y Drepana lacertinaria L.; en ambos casos la feromona generalista (9Z, 11E)-tetradecadienal (Reed and Chisholm, 1985; Arn et al., 1986). Es interesante indicar, además, que se ha sugerido actividad feromonal para Catocala grynea Cramer (Oehlke, 2004). 


\section{Conclusiones}

Los resultados anteriores permiten sugerir una doble hipótesis: por un lado la eventual existencia de diferencias de palatabilidad y/o calidad nutricional, para defoliadores tempranos, entre hojas con distinto perfil de emisión de monoterpenos; por otro, la posible influencia de la calidad alimenticia de hojas de distintos perfiles de emisión en el éxito de la actividad feromonal de ciertas especies. Hay que considerar, no obstante, que los precursores de monoterpenos derivan de la actividad fotosintética, y que el estrés ambiental y biótico induce cambios fisiológicos y bioquímicos en las plantas que afectan la emisión de monoterpenos (Niinemets et al., 2002; Lavoir et al., 2011). Por lo tanto, sería interesante estudiar con más detalle si la constitución genética - que determina diferentes perfiles de emisión- también determina diferencias en la cantidad y/o calidad del alimento para especies defoliadoras. Sería interesante, además, estudiar la eventual influencia que el estrés ambiental y biótico podría tener en esta relación, al condicionar la fisiología de los árboles.

\section{Bibliografía}

Alejano, R.; Domingo, J.M.; Fernández, M.; (coords) 2011. Manual para la gestión sostenible de las dehesas andaluzas. Foro para la Defensa y Conservación de la Dehesa "Encinal". Universidad de Huelva.

Ando, T.; 2019. List of lepidopteran sex pheromones and attractants. Recurso online: https://lepipheromone.sakura.ne.jp/index_eng.html

Antonietty, C.A.; 2013. Diseño de un plan de manejo integrado para Tortrix viridana L. (Lepidoptera: Tortricidae). Docthoral Thesis, Universidad de Sevilla.

Arn, H.; Tóth, M.; Priesner, E.; 1986. List of sex pheromones of Lepidoptera and related attractants. OILB-SROP / IOBC-WPRS, Paris, pp. 123.

Bowsher, C.; Steer, M.; Tobin, A.; 2008. Plant Biochemistry. Garland Science. New York: Taylor \& Francis Group . 446 p.

Branco, M.; Ramos, P.; 2009. Coping with pests and diseases. In: Aronson, J.; Pereira, J.S.; Pausas, J.G.; (eds.), Cork oak woodlands on the edge: ecology, adaptive management, and restoration. Island Press, Washington, pp 103-111.

Carrasco, D.; Larsson, M.C.; Anderson, P.; 2015. Insect host plant selection in complex environments. Curr. Opin. Insect Sci. 8: 1-7. https://doi.org/10.1016/j.cois.2015.01.014

Conchou, L.; Lucas, P; Meslin, C.; Proffit, M.; Staudt, M.; Renou, M.; 2019. Insect odorscapes: from plant volatiles to natural olfactory scenes. Front. Physiol. https://doi. org/10.1016/j.cois.2015.01.014

Dicke, M.; Baldwin, I.T.; 2010. The evolutionary context for herbivore-induced plant volatiles: beyond the 'cry for help'. Trends Plant Sci. 15:167-75. https://doi.org/10.10 16/j.tplants.2009.12.002

Extremera, F.M.; Cobo, A.; Pérez, M.C.; Pérez-Guerrero, S.; Vargas-Osuna, E.; 2004. El complejo de lepidópteros defoliadores de Quercus en la provincia de Córdoba. Bol. San. Veg. Plagas 30: 203-209. 
Frerot, B.; Renou, M.; Gallois, M.; and Descoins, C.; 1983. A sex attractant for Archips xylosteana L. (Lepid., Tortricidae, Tortricinae). Agronomie 3: 173-178. https://doi. org/10.1051/agro:19830209

Gols, R.; 2012. Direct and indirect chemical defences against insects in a multitrophic framework: plant chemical defences againstinsects. Plant Cell Environ. 37: 1741-1752. https://doi.org/10.1111/pce.12318

Granados, C.; Ramírez, D.; Sánchez, I.; López, G.; Vázquez, E.; 2000. Defoliadores de encinar en el Andévalo Occidental de la provincia de Huelva. Comparación entre dos situaciones puntuales: el período 1985-1988 y el año 2000. Póster presentado al Congreso Forestal Español, Granada 2000.

Grote, R.; Monson, R.; Niinemets, Ü.; 2013. Leaf-level models of constitutive and stress-driven volatile organic compound emissions. In: Niinemets, Ü.; Monson, R.K.; (eds.), Biology, controls and models of tree volatile organic compound emission. Springer Netherlands, Dortrecht. pp. 315-355. https://doi.org/10.1007/978-94-007-6606-8_12

Henneken, J.; Goodger, J.Q.D.; Jones, T.M.; Elgar, M.A.; 2017. Diet-mediated pheromones and signature mixtures can enforce signal reliability. Front. Ecol. Evol. 4: 145. https://doi.org/10.3389/fevo.2016.00145

Ivashov, A.V; Boyko, G.E.; Simchuk, A.P.; 2002. The role of host plant phenology in the evelopment of the oak leafroller moth, Tortrix viridana L. (Lepidoptera: Tortricidae). For. Ecol. Manage. 157:7-14. https://doi.org/10.1016/S0378-1127(00)00652-6

Kigathi, R.N.; Unsicker, S.B.; Reichelt, M.; Kesselmeier, J.; Gershenzon, J.; Weisser, W.W.: 2009. Emission of volatile organic compounds after herbivory from Trifolium pratense (L.) under laboratory and field conditions. J. Chem. Ecol. 35: 1335-1348. https://doi.org/10.1007/s10886-009-9716-3

Kneeshaw, D.; Sturtevant, B.R.; Cooke, B.; Work, T.; Pureswaran, D.; DeGrandpre, L.; MacLean, D.; 2015. Insect disturbances in forest ecosystems. In: Peh, K.S.-H., Corlett, R.T., Bergeron, Y. (eds.), Routledge handbook of forest ecology. Taylor \& Francis Group, New York, NY.

Lavoir, A.V.; Duffet, C.; Mouillot, F.; Rambal, S.; Ratte, J.P.; Schnitzler, J.P.; Staudt, M.; 2011. Scaling-up leaf monoterpene emissions from a water limited Quercus ilex woodland. Atmos. Environ. 45: 2888-2897. https://doi.org/10.1016/j.atmosenv.2011.02. 005

Li, J.; Valimaki, S.; Shi, J.; Zong, S.; Luo, Y.; Heliovaara, K.; 2012. Attraction of the gypsy moth to volatile organic compounds (VOCs) of damaged dahurian larch. Z. Naturforsch. 67c: 437-444. https://doi.org/10.1515/znc-2012-7-812

Luciano, P.; Roversi, P.; 2001. Oak defoliators in Italy. Industria Grafica Poddighe s.r.1., Sassari, Italy.

Magnoler, A.; Cambini, A.; 1970. Effects of artificial defoliation on the growth of cork oak. For. Sci. 16: 364-366.

López-Pantoja, G.; Domínguez, L.; Sánchez-Osorio, I.; 2008. Mark-recapture estimates of the survival and recapture rates of Cerambyx welensii Küster (Coleoptera Cerambycidae) in a cork oak dehesa in Huelva (Spain). Cent. Eur. J. Biol. 3: 431-441. https:// doi.org/10.2478/s11535-008-0044-3

Monreal, J.A.; Salvador, D.; Mansilla, J.; 1992. Contribución al conocimiento de los insectos defoliadores de la encina (Q. ilex L.), en la provincia de Albacete. Bol. San. Veg. Plagas 18: 395-405.

Niinemets, U.; Seufert, G.; Steinbrecher, R.; Tenhunen, J.D.; 2002. A model coupling foliar mo- 
noterpene emissions to leaf photosynthetic characteristics in Mediterranean evergreen Quercus species. New Phytol. 153: 257-275. https://doi.org/10.1046/j.00 28-646X.2001.00324.x

Oehlke, B.; 2004. Catocala grynea. Recurso online. http://www.silkmoths.bizland.com /Catocala/catgrynea.htm

Oksanen, F.; Blanchet, G.; Kindt, R. et al.; 2015. vegan: Community Ecology Package. R package version 2.2-1. http://CRAN.R-project.org/package=vegan

Pearse, I.S.; Gee, W.S.; Beck, J.J.; 2013. Headspace Volatiles from 52 oak species advertise induction, species identity, and evolution, but not Defense. J. Chem. Ecol. 39: 90- 100. https://doi.org/10.1007/s10886-012-0224-5

Pereira, P.; Godinho, C.; Roque, I.; Marques, A.; Branco, M.; Rabaça, J.E.; 2012. Time to rethink the management intensity in a Mediterranean oak woodland: the response of insectivorous birds and leaf-chewing defoliators as key groups in the forest ecosystem. Ann. For. Sci. 71: 25-32. https://doi.org/10.1007/s13595-012-0227-y

Pio, C.A.; Silva, P.A.; Cerqueira, M.A.; Nunes, T.V.; 2005. Diurnal and seasonal emissions of volatile organic compounds from cork oak (Quercus suber) trees. Atmos. Environ. 39: 1817-1827. https://doi.org/10.1016/j.atmosenv.2004.11.018

R Development Core Team ("R- 3.1.0”) (2014) R, A language and environment for statistical computing. R Foundation for Statistical Computing. Vienna, Austria. http:// www.R-project.org

Reed, D.W.; Chisholm, M.D.; 1985. Attraction of moth species of Tortricidae, Gelechiidae, Geometridae, Drepanidae, Pyralidae, and Gracillariidae families to field traps baited with conjugated dienes. J. Chem. Ecol. 11: 1645-1657. https://doi.org/10.1007/BF01 012118

San Miguel, A.; 1994. La dehesa española. Origen, tipología, caracteristicas y gestión. Fundación Conde del Valle de Salazar, Madrid.

Sánchez-Herrera, F.; Soria, S.; 1987. La problemática del seguimiento y control de lepidópteros nocivos del encinar, especial referencia al encinar adehesado madrileño. Bol. San. Veg. Plagas 13: 213-224.

Sánchez-Osorio, I.; López-Pantoja, L.; Tapias, R.; Pareja-Sánchez, E.; Domínguez, L.; 2019. Monoterpene emission of Quercus suber L. highly infested by Cerambyx welensii Küster Ann. For. Sci. 89. https://doi.org/10.1007/s13595-019-0879-y

Solla, A.; Milanovic, S.; Gallardos, A.; Bueno, A.; Corcobado, T.; Cáceres, Y.; Morcuende, D.; Quesada, A.; Moreno, G.; Pulido, F; 2016. Genetic determination of tannins and herbivore resistance in Quercus ilex. Tree Gen. Genom. 12: 117. https://doi.org/ 10.1007/s11295016-1069-9

Soria, S.; 1988. Relación de lepidópteros paleárticos defoliadores del género Quercus L. Bol. San. Veg. Plagas 14: 11-26.

Srinivasan, R.; 2019. Introduction: host plant choice and feeding ecology of insects. Entomol. Exp. Appl. 167: 288-291. https://doi.org/10.1111/eea.12787

Staudt, M.; Jackson, B.; El-Aouni, H.; Buatois, B.; Lacroze, J.P.; Poëssel, J.L.; Sauge, M.H.; 2010. Volatile organic compound emissions induced by the aphid Myzus persicae differ among resistant and susceptible peach cultivars and a wild relative. Tree Physiol. 30:1320-34. https://doi.org/10.1093/treephys/tpq072

Staudt, M.; Mir, C.; Joffre, R.; Rambal, S.; Bonin, A.; Landais, D.; Lumaret, R.; 2004. Isoprenoid emission of Quercus spp. (Q. suber and $Q$. ilex) in mixed stands contrasting in interspecific genetic introgression. New Phytol. 163: 573-584. https://doi.org/10.1 111/j.14698137.2004.01140.x 
Summerville, K.; Marquis, R.; 2016. Comparing the responses of larval and adult lepidopteran communities to timber harvest using long-term, landscape-scale studies in oak-hickory forests. Forest. Ecol. Manag. 387: 64-72. https://doi.org/10.1016/j.foreco.20 16.08.050

Teodorescu, I.; Simionescu, A.; 1994. Dynamics of defoliating Lepidoptera attacks and the control measures in Romania deciduous forests, 1953-1990. Ambio. 23: 260-266.

Torrent, J.A.; 1963. Montaneras en los últimos diez años (1953-62). Bol. Serv. Plag. For. 11: $73-77$.

Thomas, F.; 2008. Recent advances in cause-effect research on oak decline in Europe. CAB. Rev. Perspect. Agric. Vet. Sci. Nutr. Nat. Resour. 3: 1-12. https://doi.org/10.10 79/PAVSNNR20083037

Tiberi, R.; Branco, M.; Bracalini, M.; Croci, F.; Panzavolta, T.; 2016. Cork oak pests: a review of insect damage and management. Ann. For. Sci. 73: 219-232. https://doi.org/ 10.1007/s13595-015-0534-1

Toimil, F.J.; 1987. Algunos lepidópteros defoliadores de la encina (Q. ilex L.) y alcornoque ( $Q$. suber L.), en la provincia de Huelva. Bol. San. Veg. Plagas 13: 331-346. 
Robles et al. | Cuad. Soc. Esp. Cienc. For. (2020) 46(1): 1-16 